\title{
Simulation-Based Design of Aircraft Electrical Power Systems
}

\author{
Tolga Kurtoglu Peter Bunus Johan De Kleer \\ Palo Alto Research Center \\ 3333 Coyote Hill Dr. Palo Alto, CA 94304 \\ kurtoglu@parc.com peter.bunus@parc.com dekleer@parc.com
}

\author{
Rahul Rai \\ California State University \\ Department of Mechanical Engineering, Fresno, CA 93740 \\ rarai@csufresno.edu
}

\begin{abstract}
Early stage design provides the greatest opportunities to explore design alternatives and perform trade studies before costly design decisions are made. The goal of this research is to develop a simulation-based framework that enables architectural analysis of complex systems during the conceptual design phase. Using this framework, design teams can systematically explore architectural design decisions during the early stage of system development prior to the selection of specific components. The analysis performed at this earliest stage of design facilitates the development of more robust and reliable system architectures. Application of the presented method to the design of a representative aerospace electrical power system (EPS) demonstrates these capabilities.
\end{abstract}

Keywords: simulation-based design; electrical power system; architectural design; concept generation

\section{Introduction}

The complexity of modern world engineered systems is growing constantly. New technologies are creating the potential for higher levels of integration and resulting systems contain a larger number of dynamically interacting components, relations among which are increasingly non-linear. This complexity, in turn, leads to unexpected behaviors and consequences, some of which have proven to be catastrophic. A key technical challenge in developing such complex sys- tems is to ensure that the individual components and technologies are reliable, effective, and low cost, resulting in turn in safe, reliable, and affordable systems.

To address these challenges, DARPA's META Program is investing in novel methods for design and verification of complex systems. The META program is specifically aimed at compressing the product development and deployment timeline by enabling model-based design and manufacturing across the complex, heterogeneous, and physically-coupled electromechanical systems. Using this design paradigm, different "component model libraries" or "physics libraries" can be interchangeably used to instantiate a given system design such that a design can be analyzed and verified entirely independently of its physical manifestation [1].

On the other hand, ensuring safety, reliability, affordability, and performance requires the incorporation of subsystem and component functionality, decisions and knowledge into the product lifecycle as early as possible. Furthermore, formal tools and methodologies need to be in place to allow design teams to formulate a clear understanding of the impact of the decisions in the early design phases.

Developed as part of the META program, this paper presents a simulation-based design framework that enables architectural analysis of complex systems during the conceptual design phase. Using this framework, design teams can systematically explore architectural design decisions during the early stage of system development prior to the selection of spe- 
cific components. The analysis performed at this earliest stage of design facilitates the development of more robust and reliable system architectures. In this paper, we describe the proposed framework and present the application of its use to the design of a representative aerospace electrical power system (EPS).

\section{Integrated System Design and Analysis Framework}

The framework is the basis for specifying system requirements, supporting design space exploration, and analyzing the performance associated with promising architectural design alternatives. To support a model-based design paradigm, the framework allows the designers to combine models from different domains into integrated system level models, and allow models of components and sub-systems to evolve throughout the design process. At the end, component models are composed into a system that achieves the intended functionality given specified requirements such as reliability, risk, and performance.

In what follows, we describe the constituent elements of the framework but first a brief overview of electrical power system design is provided.

\subsection{Electrical Power System Design}

An electrical power system is designed to deliver power to select loads, which in an aerospace vehicle would include subsystems such as the avionics, propulsion, life support, and thermal management systems. The EPS is required to provide basic functionality common to many aerospace applications: power storage, power distribution, and operation of loads [2].

An EPS system was originally designed by one of the co-authors using a failure-based design methodology at the early concept design phase [3]. Using this function-based design approach, several critical elements were identified and incorporated into the final design and realization of the system.

In the current realization of the system, which is illustrated in Fig. 1, the power storage consists of one or multiple battery modules, which are used to store energy for the operation of the loads. Any of the battery modules can be used to power any number of loads in the system. This requires the EPS to have basic redundancy and reconfiguration capability. Electromechanical relays or other electrical actuators can be used to route the power from the batteries to the loads. In addition, circuit breakers are added to the design at various points in the distribution net- work to prevent overcurrents from causing unintended damage to the system components. Moreover, a sensor suite is designed in to allow monitoring of voltages, currents, temperatures, switch positions, etc. and to provide an integrated health management functionality. (More information on the existing electrical power system can be found in [2].

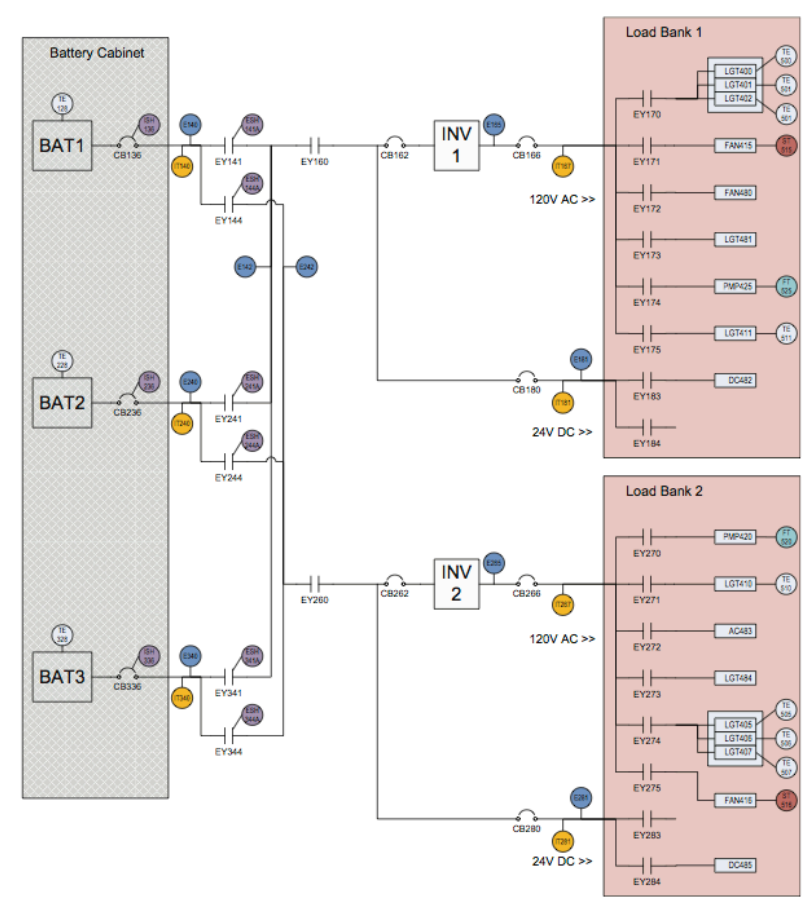

Fig. 1. The schematic of the existing electrical power system design architecture

\subsection{A Modelica Library for an Aircraft EPS}

In this paper, we extend our previous, function-based analysis of the EPS system and explore how well different EPS architectures meet specified reliability, risk, and performance requirements.

The building blocks within the presented modelbased design environment are component objects consisting of a set of configurational, and behavioral models, component interfaces, and relationships between them. Accordingly, we build a Modelicabased [4] component model library, which will present the designers a set of available reconfigurable models, and include physical artifacts such as batteries, actuators, electrical switches, etc. The models are stored in an EPS Design Repository. For each component of the EPS Modelica library the nominal behavior was modeled and augmented with the relevant failure modes. A list of operating and possible failure modes of the EPS Modelica library components is depicted in Table $\mathbf{1}$. 
Table 1. A list of components of the EPS Modelica libraries and the associated nominal operating and failure modes.

\begin{tabular}{|c|c|c|}
\hline Model Element & $\begin{array}{l}\text { Element } \\
\text { Type }\end{array}$ & $\begin{array}{l}\text { Operating and } \\
\text { fault modes }\end{array}$ \\
\hline Battery & Source & $\begin{array}{l}\text { Nominal, } \\
\text { AbruptParasiti- } \\
\text { cLoad }\end{array}$ \\
\hline CircuitBreaker & $\begin{array}{l}\text { Electrical } \\
\text { Circuit } \\
\text { Breaker }\end{array}$ & $\begin{array}{l}\text { Nominal, Tripped, } \\
\text { FailedOpen, Stuck- } \\
\text { Closed }\end{array}$ \\
\hline Relay & $\begin{array}{l}\text { Electome- } \\
\text { chanical Re- } \\
\text { lay }\end{array}$ & $\begin{array}{l}\text { NominalClosed, } \\
\text { NominalOpen, } \\
\text { StuckOpen }\end{array}$ \\
\hline Inverter & $\begin{array}{l}\text { Electrical } \\
\text { Inverter }\end{array}$ & $\begin{array}{l}\text { NominalOn, Nomi- } \\
\text { nalOff, FailedOff }\end{array}$ \\
\hline $\begin{array}{l}\text { Temperature- } \\
\text { Sensor }\end{array}$ & $\begin{array}{l}\text { Temperature } \\
\text { Sensor }\end{array}$ & $\begin{array}{l}\text { Nominal, Drift, } \\
\text { Offset, Intermitten- } \\
\text { tOffset, Stuck }\end{array}$ \\
\hline $\begin{array}{l}\text { DCCurrent- } \\
\text { Tranmitter }\end{array}$ & $\begin{array}{l}\text { DC Current } \\
\text { Transmitter } \\
\text { (50A Max) } \\
\end{array}$ & $\begin{array}{l}\text { Nominal, Drift, } \\
\text { Offset, Intermitten- } \\
\text { tOffset, Stuck }\end{array}$ \\
\hline $\begin{array}{l}\text { DCVoltageSen- } \\
\text { sor }\end{array}$ & $\begin{array}{l}\text { DC Voltage } \\
\text { Sensor } 10 \mathrm{HZ}\end{array}$ & $\begin{array}{l}\text { Nominal, Drift, } \\
\text { Offset, Intermitten- } \\
\text { tOffset, Stuck }\end{array}$ \\
\hline PositionSensor & $\begin{array}{l}\text { Actuator Posi- } \\
\text { tion Sensor } \\
10 \mathrm{HZ}\end{array}$ & Nominal, Stuck \\
\hline $\begin{array}{l}\text { ACResistor } \\
\text { DCResistor }\end{array}$ & $\begin{array}{l}\mathrm{AC} \text { and } \mathrm{DC} \\
\text { Resistors }\end{array}$ & $\begin{array}{l}\text { Nominal, FailedOff, } \\
\text { IntermittentResis- } \\
\text { tanceOffset, Resis- } \\
\text { tanceDrift, Resis- } \\
\text { tanceOffset }\end{array}$ \\
\hline LargeFan & LargeFan & $\begin{array}{l}\text { Nominal, Over- } \\
\text { Speed, UnderSpeed, } \\
\text { FailedOff }\end{array}$ \\
\hline LightBulb & $\begin{array}{l}\text { 25W Light } \\
\text { Bulb }\end{array}$ & Nominal, FailedOff \\
\hline WaterPump & Water Pump & $\begin{array}{l}\text { Nominal, } \\
\text { FlowRestricted, } \\
\text { FailedOff }\end{array}$ \\
\hline
\end{tabular}

For several components, models with different levels of detail have been created. For example, the Inverter component created Modelica models are ranging from very simple models that describe only the $\mathrm{AC} / \mathrm{DC}$ power balance equation to models containing complicated electrical schematics including semiconductor components from the Electrical Standard Modelica Library. The reason for creating models of the same component with different levels of details was to compare how our proposed architecture analysis methods performs in very early stages of the conceptual analysis, when not so much details are be available, to later stages when more details are added to the component models.
The nominal and the fault modes behavior of the Modelica EPS Library components have been validated by comparing the simulation behavior of two test models with measurements and sensor data from the Advanced Diagnostics and Prognostics testbed called ADAPT located at the NASA Ames Research Center [5,6]. (The ADAPT system consists of a controlled and monitored environment where faults can be injected into the system in a controlled manner and the performance of the test article is carefully monitored.)

The first test Modelica model, called the ADAPT Tier 1 (ADAPT Lite) model, depicted in Fig 2, contains a battery connected through a series of circuit breakers and relays to an inverter, and several loads consisting of a large fan, a DC resistor and $\mathrm{AC}$ resistor. The rotation speed of the fan is measured by a speed transmitter component. A series of four AC or DC voltage sensors and three current transmitters measure the voltage and current in different probing points of the circuit. The circuit breakers can be commanded externally to be closed or open and their position is monitored with the help of a position sensor connected to them.

The second EPS model (ADAPT Tier2) that has been tested and built in Modelica is depicted in Fig 3 and it is equivalent to the schematic represented in Fig 1. In this model the ADAPT Tier 2 EPS supplies power to five critical load functions and four noncritical loads distributed in two load banks. The battery cabinet unit contains three battery packs and several relays that control the connections between the load bank and the batteries. Similarly to the ADAPT Lite model, the testbed is controlled by a number of relays and monitored by a large set of sensors.

The ADAPT Tier 1 model has been validated against 39 experiments while the ADAPT Tie 2 model has been validated against 33 experiments simulating nominal and faulty behavior of the EPS.

Since each component contains a description of the failure behavior besides the description of the nominal behavior, by systematically selecting a certain state of the system and inducing faults in the components, we were able to observe the effects of those faults on the system and automatically build a Failure Model and Effect Analysis (FMEA) table from the model. 


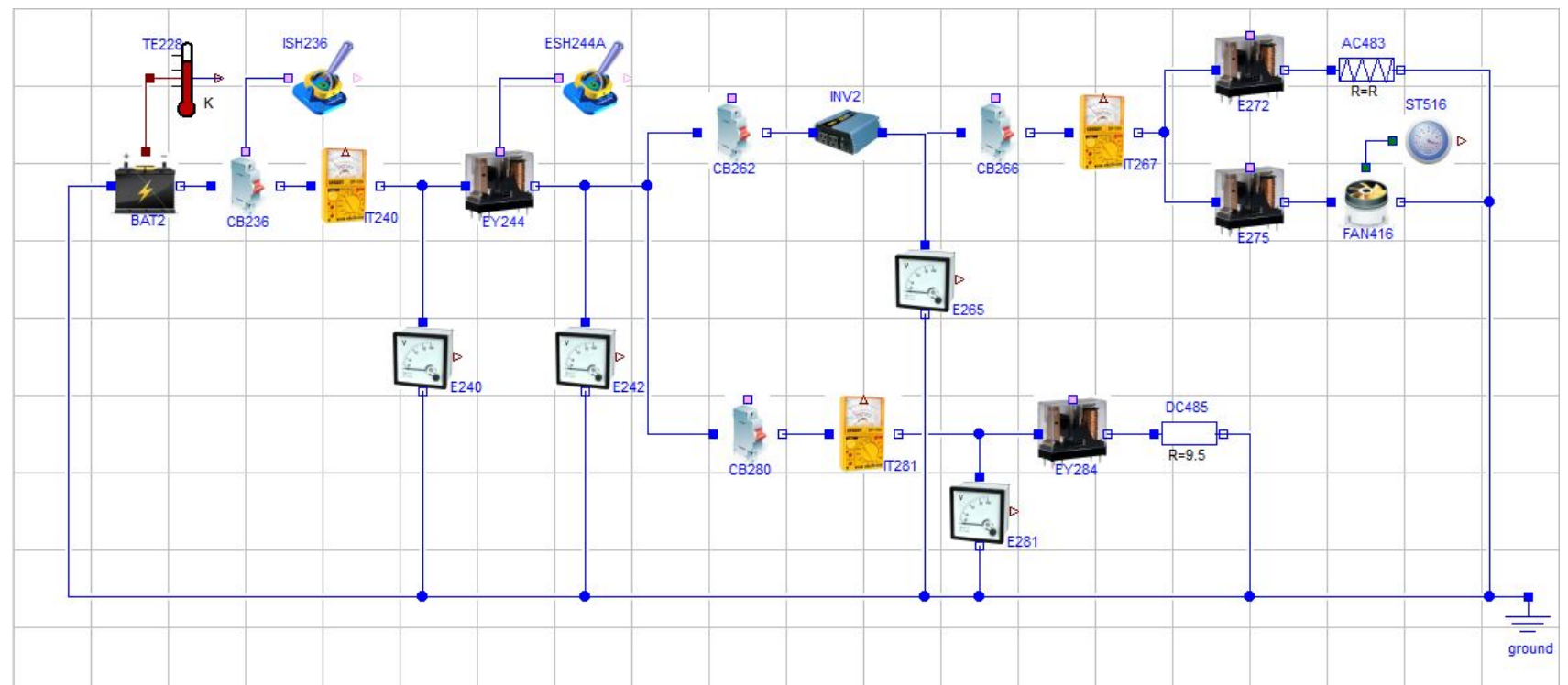

Fig. 2. The Modelica model of simple EPS system (ADAPT Tier 1).

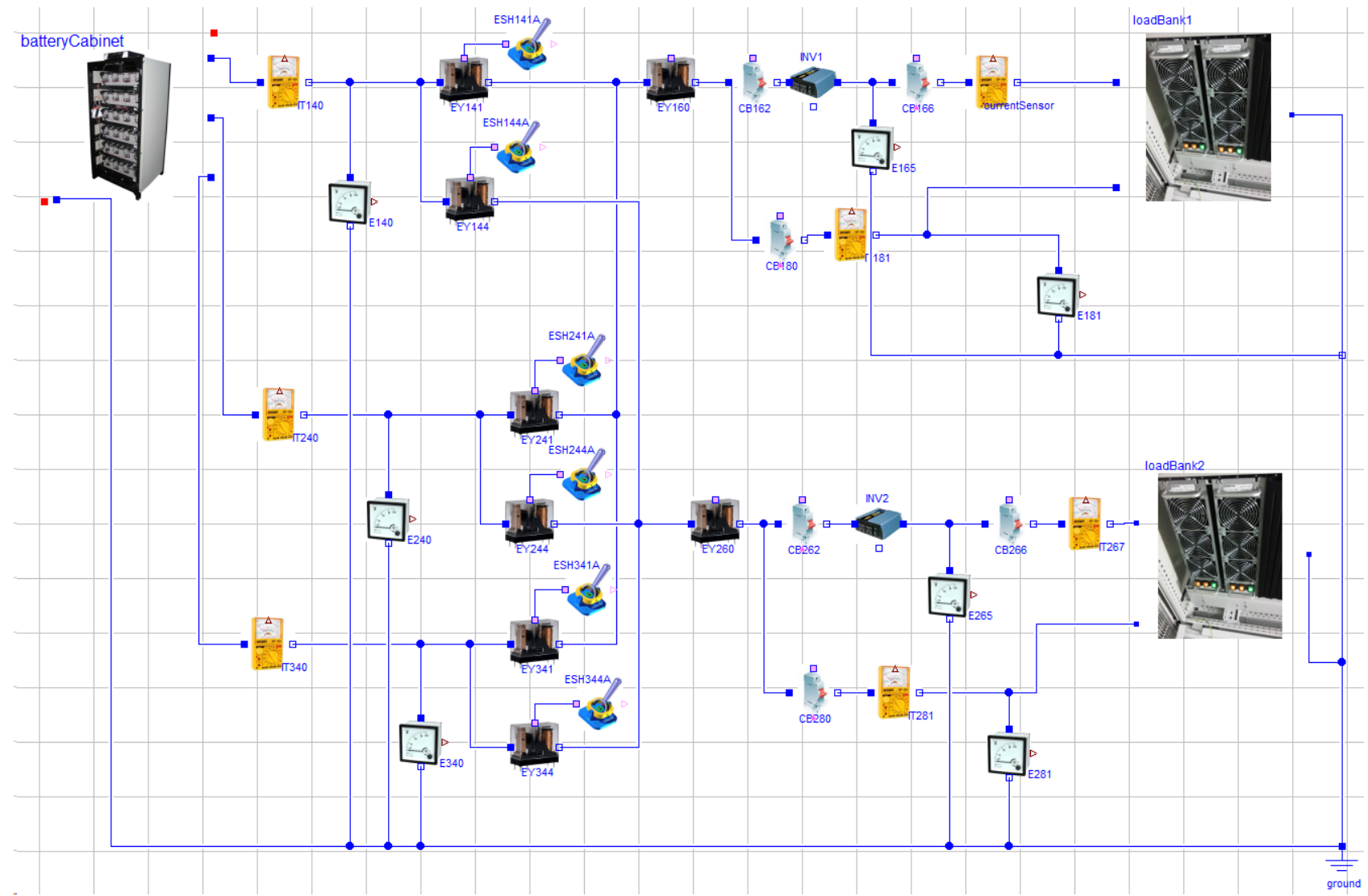

Fig. 3. The schematic of the existing electrical power system design architecture.

\subsection{Design Space Exploration}

This section examines the means of exploring the design space defined by combinations of generic EPS components. Feasible EPS candidate architectures are generated by a generative grammar based design space exploration technique. This generative technique takes user specified EPS loads as input and satisfies system-level configuration requirements to generate feasible EPS candidate architectures. The component model library serves as the backbone of the proposed design space exploration approach. Using the models in the model library as building blocks, this generative graph grammar based technique configures "correct by construction" EPS ar- 


\begin{abstract}
b. A battery relay shall be installed in each battery circuit to enable the flight crew to isolate the battery from the rest of the electric subsystem. The battery relay shall be controlled by a crew station battery switch. Any circuits which must remain connected to the battery with the battery switch OFF shall be connected directly to the battery through suitable fuses or circuit breakers.
\end{abstract}

Fig. 4. An architectural design requirement that is used in derivation of a design grammar

chitectures that can be further studied by means of a simulation-based analysis.

Graph grammar based configuration approach uses graph as representation scheme. These approaches capture the transitions or the production rules for creating a solution, as opposed to storing the solutions themselves. Accordingly, a configuration's development from its inception to its final configuration is considered as a series of graph modifications. The initial specification can be represented as a simple graph in which the desired inputs and outputs are cast as arcs and nodes of the to-be-designed artifact. From this initial specification, the design process can be viewed as a progression of graph transformations that lead to the final configuration [7]. Recently, engineering design researchers have discovered that graph grammars provide a flexible yet ideally structured approach to the creation of complex engineering systems [8-10]. This interpretation of the design process makes graph grammars very suitable for computationally modeling the open-ended nature of conceptual design, where designers explore various ideas, decisions, and modifications to previous designs to arrive at feasible solutions.

Generating feasible EPS architecture using graph grammar based configuration approach is a two-step process: In the first step, we have developed an EPS system design grammar to encode design rules for constructing electrical power system architectures. For EPS, we have developed a 14-rule graph grammar that defines ways to generate feasible EPS architectures from multiple EPS requirement documents [11-15]. The rules are established prior to the design process and capture architectural design considerations that are inherent to the EPS design problem. One such EPS design requirement is shown in Figure
4. Similar design rules govern the mapping of functional requirements to components, or the physical compatibility between EPS components. Moreover, the graph grammar rules can be formulated in such a way that the final solution meets the constraints of the problem. The knowledge captured in the rules offer the option of exploring the design alternatives as well as automating the design generation process. Specifically, the developed design grammar encodes how specific system requirements can be embodied by selecting components from a full spectrum of electromechanical components represented in the component library.

In the second step, the graph transformation systems, or graph grammars, is invoked algebraically. Algebraic graph transformation methods rigorously define mathematical operations such as addition and intersection of graphs. A typical graph grammar rule is compromised of a left-hand side (LHS) and a right-hand side (RHS) (Figure 5). The LHS contains the conditions, upon which the applicability of a rule is determined. Accordingly, the LHS describes the state of the graph for a particular rule to be applicable. The RHS, on the other hand, contains the resulting graph transformation. It describes the new state of the graph after the application of the rule. By simply executing different combinations of grammar rules, a variety of feasible EPS architectures can easily be generated including the architecture of the ADAPT test bed shown in Figure 1.

A partial sequence of application of different EPS grammar rules to create a feasible EPS architecture is shown in Figure 6. In order to generate a feasible EPS architecture the approach starts with a seed graph. The seed graph for EPS design space exploration is graph based representation of three main

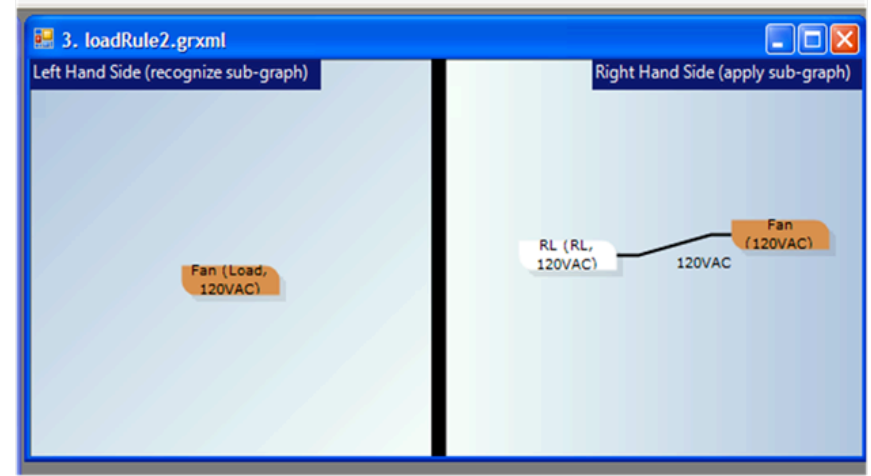

Figure 5. A graph grammar rule for EPS architecture generation 


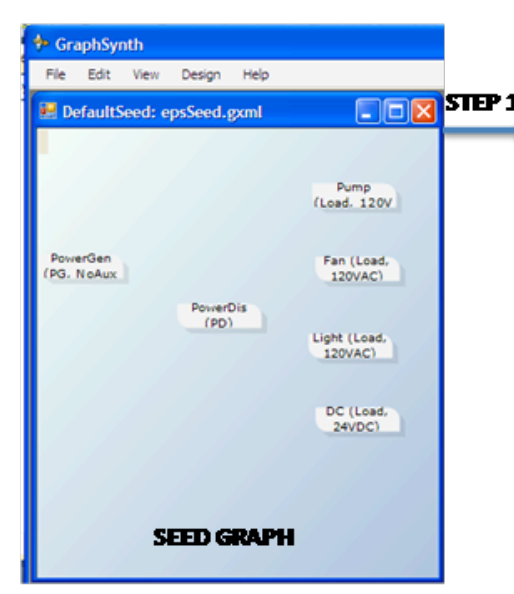

(a)

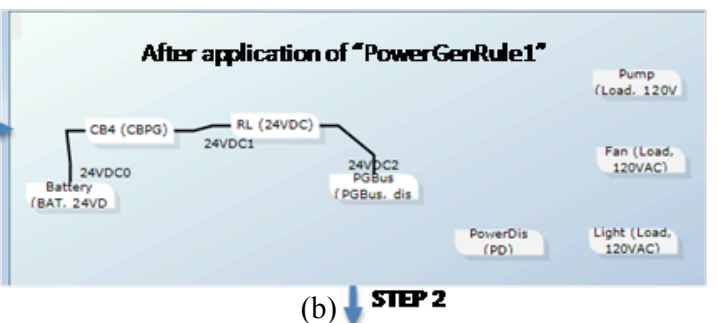

(b) $\backslash$ STEP 2

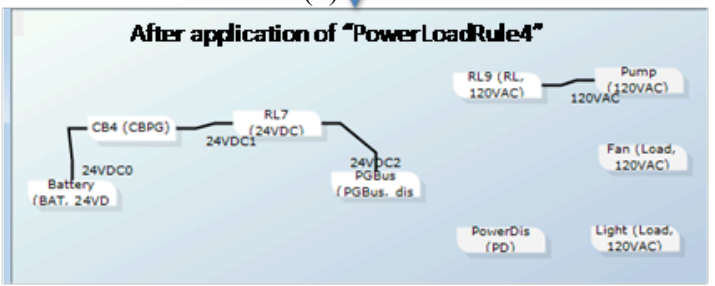

(c)

Figure 6. Application of graph grammar rules to create an EPS architecture (a) seed graph (b) modified seed graph after step 1 (c) modified seed graph after step 2

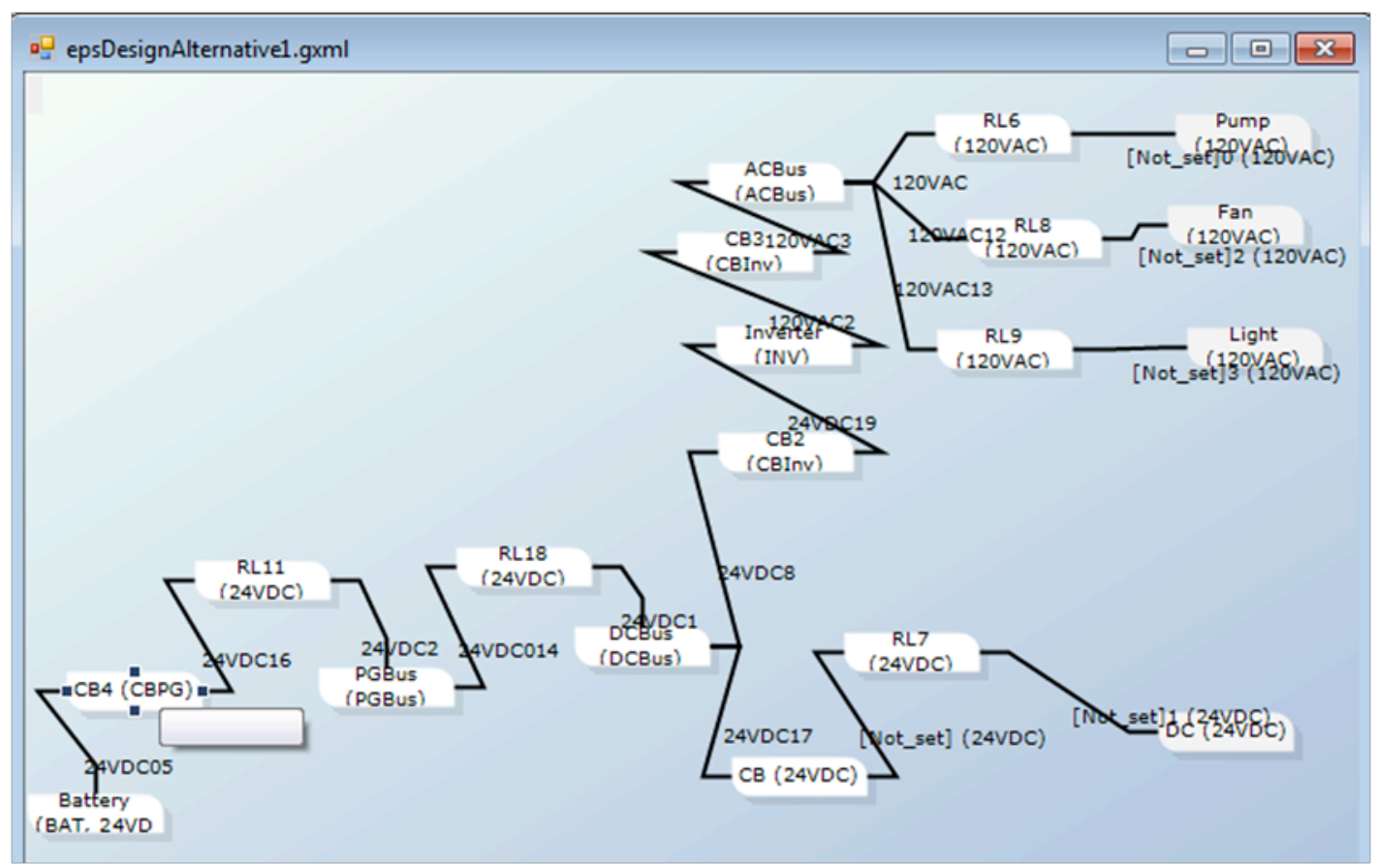

Figure 7. A feasible EPS architecture generated by generative graph grammar

subsystems of EPS namely Power Generation, Power Distribution, and Loads (Figure 6a). The loads to be supported by the EPS have to be specified and are an input to the overall process. At this stage it is recognized that 11 rules are applicable. Out of the applicable rules, rule 11 is chosen and applied by a designer (or an automated computational process). This result in a new graph in the right hand side (RHS) of step one (Figure 6b). This RHS in step one becomes LHS in step two. After this stage the process of recognize, choose and apply is invoked in an iterative manner resulting in a new LHS and RHS at each step. A fea- sible EPS architecture generated at the end of this process is depicted in Figure 7.

As shown with this example, generative graph grammar gives an EPS architect the ability to systematically explore a large number of alternative EPS architectures that meet a given set of design constraints and objectives. In the next section, we describe how these design alternatives are evaluated. 


\subsection{Simulation-Based Performance Analysis}

Let us consider the following set of safety, functional and performance requirements imposed on the EPS system.

Safety Requirements imposed in the architecture:

- "AFGS-87219A: A battery relay shall be installed in each battery circuit to enable the flight crew to isolate the battery from the rest of the electric subsystem."

- "MIL-STD 7080: A switch or relay shall be connected in series with the circuit breaker when a switching capability is required for a circuit protected by a circuit breaker."

Functional Requirements:

- "MIL-STD 704F: "Loads should not introduce excessive current distortion such that other EPS functionality is effected."

Performance Requirements:

- "MIL-STD-1275D: The [28 VDC electrical power system] circuit steady-state voltage shall be between 25 VDC and 30 VDC."

- "MIL-STD-1275D: The rotational speed of cooling fan system should be between 765 and 900 rpm.

As it was described in Section 2.3 feasible EPS candidate architectures are generated by a generative grammar based design space exploration technique. The graph grammar configuration approach is able to impose the safety requirements detailed above by encoding the safety requirements in graph grammar rules that are applied by the transformation system resulting in an architecture that is correct by definition. The Modelica model of a simple EPS system, (ADAPT Tier2) depicted in Fig 2 satisfy both safety requirements: the relay EY244 will isolate the battery from the rest of the electrical circuit (the first safety requirement $A F G S-87219 A$ ) while the circuit breaker-relay pairs (CB236-EY244, CB266-E272, CB266-EY275, CB280-EY284) will satisfy the second requirement from MIL-STD 7080.

The functional and performance requirements, on the other hand, are verified by simulation. Simulationbased design methods require the capability of specifying detailed input design parameters and using them to obtain a model response. Accordingly, we use a simulation process which allows system designers use to account for the effects of variability in the input and design parameters on the model response, thereby incorporating uncertainty into the design process. In this research, we use a sampling based technique to perform a simulation-based analysis of system performance. This analysis provides a means to estimate the probability of system response and assess how well a candidate system design meets its requirements.

For example, in the ADAPT Tier 1 EPS the designer has the choice of using a Xantrex Prosine 1000 Invertor or a Xantrex Freedom HW 1000 Invertor. Both variants will satisfy the safety requirements imposed on the architecture. The Xatrex Prosine 1000 Watt Inverter has a peak efficiency of $90 \%$ while the output voltage (over full load and battery voltage range) is around $120 \mathrm{Vac}-10 \% /+4 \%$. The range of the output voltage for this type of invertor can be defined as a triangular probability distribution function. The output voltage histogram for 200 samples is depicted in Figure 8.

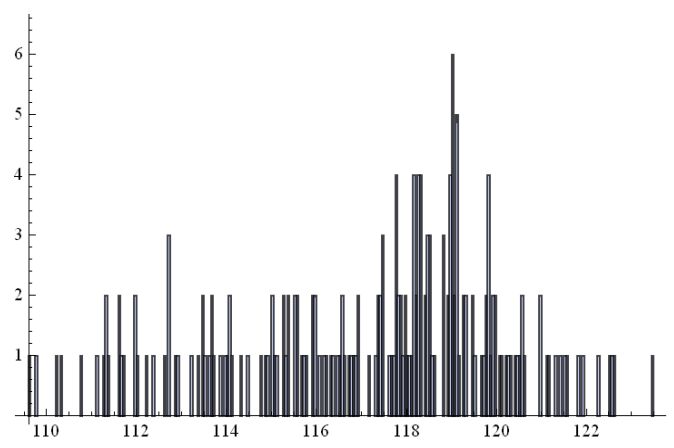

Figure 8. The Xantrex Prosine 1000 Invertor output voltage histogram.

We perform a simulation based performance analysis and we compute the rotational speed of the cooling fan for different output voltages of the inverter. The histogram of the rotational speed of the cooling fan shows that using a Xantrex Prosine 1000 Invertor is a valid architecture, which satisfies the performance requirement that the speed of the cooling fan should be between 765 and $900 \mathrm{rpm}$.

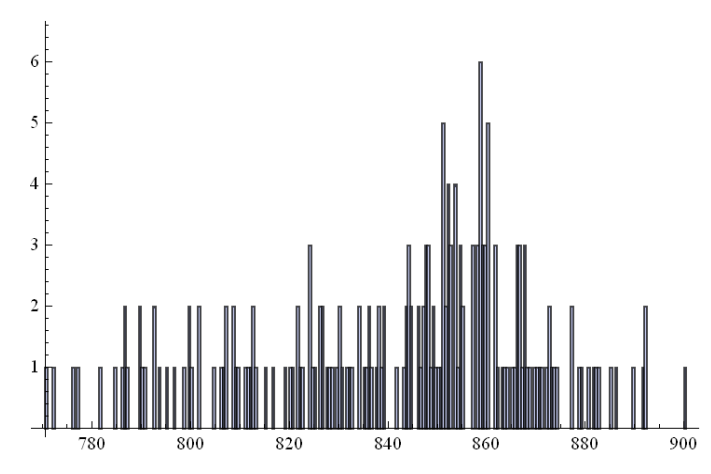

Figure 9. The histogram of the rotational speed of the cooling fan when a Xantrex Prosine 1000 invertor is used. 
The Xantrex Freedom HW 1000 Invertor has slightly different characteristics: a peak efficiency of $83 \%$ and an output voltage (over full load and battery voltage range) around $115 \mathrm{Vac}+/-10 \mathrm{Vac}$ that can be also approximated as a triangular distribution function. The histogram of the output voltage is shown in Figure 10.

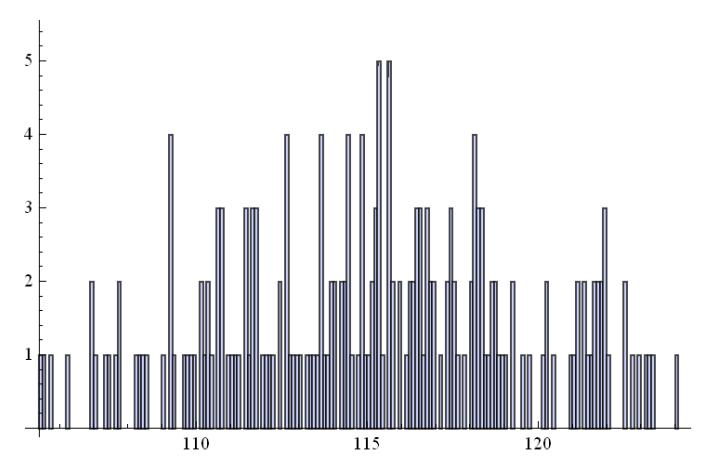

Figure 10. The Xantrex Freedom HW 1000 Invertor output voltage histogram.

A performance based simulation shows that the rotational speed of the cooling fan can sometimes drop below $765 \mathrm{rpm}$ for certain AC output voltages of the Xantrex Freedom HW 1000 Invertor (see Figure 11).

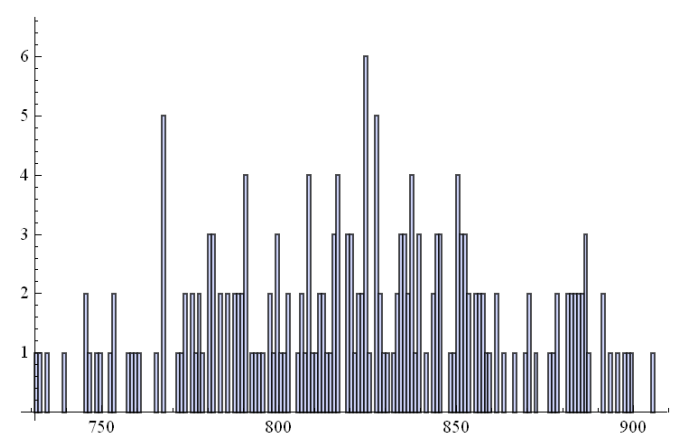

Figure 11. The histogram of the rotational speed of the cooling fan when a Xantrex Freedom HW 1000 invertor is used.

Since the performance requirements of an EPS architecture using a Xantrex Freedom 100 Invertor are not met, this architecture can be discarded from the list of alternative EPS designs.

\section{Conclusions}

We have outlined a framework for simulation-based design that integrates architectural synthesis and analysis of complex systems during the conceptual design phase. The incorporation of automated design space exploration methods with Modelica broadens the scope of the capabilities of the language, and enables it to support architectural trade studies before costly design decisions are made. In this paper, we presented preliminary results of our study. In the future, we plan to fully integrate and automate the architectural synthesis and analysis approaches described in this paper.

\section{References}

[1] Defense Advanced Research Projects Agency (DARPA), Tactical Technology Office (TTO) META-II, BAA-10-59, 2010.

[2] S. Poll, A. Patterson-Hine, J. Camisa, D. Garcia, and D. Hall, "Advanced Diagnostics and Prognostics Testbed," in 18th International Workshop on Principles of Diagnosis (DX-07) Nashville, TN, 2007.

[3] T. Kurtoglu, Jensen, D., Tumer I.Y., "A Functional Failure Reasoning Methodology for Evaluation of Conceptual System Architectures", Journal of Research in Engineering Design, published online, January 31, 2010.

[4] Modelica Language, www.modelica.org

[5] Poll Scott, Ann Patterson-Hine, Joe Camisa, David Garcia, David Hall, Charles Lee, Ole J. Mengshoel, Christian Neukom, David Nishikawa, John Ossenfort, Adam Sweet, Serge Yentus, Indranil Roychoudhury, Matthew Daigle, Gautam Biswas, and Xenofon Koutsoukos. (2007). "Advanced Diagnostics and Prognostics Testbed." In Proceedings of the International Workshop on Principles of Diagnosis (DX-07). (Nashville, TN, May 2007, 2007).

[6] NASA Ames Research Center (2006) "Advanced Diagnostics and Prognostics Testbed (ADAPT) System Description, Operations, and Safety Manual," February, 2006.

[7] Cagan, J., 2001, "Engineering Shape Grammars," Formal Engineering Design Synthesis, Antonsson, E. K., and J. Cagan, eds., Cambridge University Press.

[8] Rai, R., Kurtoglu, T., and Campbell, M., 2009 ,"Stochastic interactive graph grammar search for conceptual design" ASME Journal of Computing and Information Sciences in Engineering (Accepted for Publication with review).

[9] Kurtoglu, T., Campbell, M., "Automated Synthesis of Electromechanical Design Configurations from Empirical Analysis of Function to Form Mapping". Journal of Engineering Design, Vol. 20 (1), Feb 2009. 
[10] Shea, K., J. Cagan, and S.J. Fenves, 1997, “A Shape Annealing Approach to Optimal Truss Design with Dynamic Grouping of Members", ASME Journal of Mechanical Design, Vol 119, No. 3, pp. 388-394.

[11] DEPARTMENT OF DEFENSE, "Aircraft electric power characteristics", MIL-STD704F, 12 March 2004.

[12] DEPARTMENT OF DEFENSE, "Air Force Specification Guide: Electrical Power Systems", Aerospace Vehicles, AFGS87219A, 30 March 1993.

[13] DEPARTMENT OF DEFENSE, "Characteristics of 28 Volt DC Electrical Systems in Military Vehicles", MIL-STD1275D, 29 August 2006.

[14] DEPARTMENT OF DEFENSE, "Selection and Instillation of Aircraft Electronic Equipment”, MIL-STD-7080, 31 May 1994

[15] DEPARTMENT OF DEFENSE, "Joint services specification guide (JSSG-2009) air vehicle subsystems", Appendix H, 30 October 1998. 